\title{
MSI1 Gene
}

National Cancer Institute

\section{Source}

National Cancer Institute. MSI1 Gene. NCI Thesaurus. Code C74471.

This gene is involved in the modulation of translation. 